\title{
El Sistema Nacional de Investigadores en México como mecanismo meritocrático de un Estado Evaluador
}

\section{The National System of Researchers in Mexico as Meritocratic Mechanism of an Evaluating State}

\section{José Alfonso Jiménez Moreno (iD}

Universidad Autónoma de Baja California.

jose.alfonso.jimenez.moreno@uabc.edu.mx

\section{Resumen}

En el presente artículo se analiza el problema del Sistema Nacional de Investigadores en México (SNI) como medio de implementación de una política meritocrática del Estado. Primero se presenta una reflexión sobre la eficiencia del SNI, considerando la evolución de la participación de los investigadores mexicanos bajo la mirada analítica de la técnica del poder. Luego se analiza su papel en el capitalismo académico como un agente del Estado Evaluador. Se concluye cuestionando al Estado Mexicano frente a su responsabilidad como soporte de desarrollo de la ciencia con respecto a su actual rol como medio regulador del poder del capitalismo académico y valorándolo como un factor de desarrollo de las condiciones necesarias para mejorar el desarrollo de la investigación.

Palabras Clave: Sistema Nacional de Investigadores, meritocracia, Estado Evaluador, poder, investigación científica.

\section{Abstract}

This paper presents the problem of the National System of Researchers (SNI) in Mexico, as a way of the Mexican State a way to implement a meritocratic policy. The analysis that is presented relies on the efficiency of the SNI, considering the evolution of the participation of Mexican researchers, under the analytical framework of the power technique; likewise, it focuses on its role as a mechanism of implementation of the policy of academic capitalism as an agent of the Evaluating State. The paper concludes by questioning the role of the Mexican State in its responsibility as a support for the development of science regarding its role as a way of regulator of the power of academic capitalism and valuing it as a factor of development of the necessary conditions to improve the development of research.

Key Words: National System of Researchers; Meritocracy; Evaluative State; Power; Scientific research.

Artículo: Recibido el 15 de enero de 2018 y aprobado 15 de octubre de 2018.

Cómo citar este artículo:

Jiménez, J.A. (2019). El Sistema Nacional de Investigadores en México como mecanismo meritocrático de un Estado Evaluador. Reflexión Politica 21 (41), pp. 81-90. DOI: https://doi.org/10.29375/01240781.2850 


\section{Introducción}

Algunas de las funciones de los académicos dentro de las universidades en todo el mundo son, entre otras, la docencia y la investigación. Actualmente se asume sin mayores conflictos que aquellas instituciones que se orientan a la mayor productividad de actividades de investigación son consideradas instituciones de calidad. Incluso, dentro de la tendencia actual de las universidades, el mecanismo más eficiente para identificar las demandas sociales a las cuales se debe orientar la investigación es el mercado, al punto que han surgido rankings que se enfocan en mostrar la competencia por la calidad (Márquez, 2010).

Adicionalmente, es indudable que, tanto en la esfera pública como en la privada, la investigación científica $^{1}$ es considerada como motor de desarrollo social y económico. Este interés para desarrollar la investigación, dada su importancia para el desarrollo, pero también por su valor como factor de competitividad, ha traído como resultado la implementación de diversas estrategias para incentivar la producción científica. Esto se observa en diferentes partes del mundo y, en lo particular, en algunos países de habla hispana como México, España, Argentina, Venezuela, Uruguay y Paraguay (Cabrero-Mendoza, 2015; Walker, 2017).

En el caso de México, a partir de 1984 el Estado estableció el Sistema Nacional de Investigadores (SNI): un programa de política pública que favorecería el desarrollo científico y a la vez fomentaría la formación de investigadores en todo el país. La incorporación de los investigadores al SNI se fundamentó en la valoración de su productividad en la investigación científica. Actualmente el SNI agrupa a los investigadores más productivos de México y ha permitido la consolidación de otros programas de política pública orientados a la mejora de la calidad de las investigaciones, como el Padrón Nacional de Posgrados de Calidad (PNPC) ${ }^{2}$ (López-Olmedo, et al., 2017). La creación del SNI suele atribuirse al interés del Estado para detener la llamada fuga de cerebros (Millán y Meza, 2015; Reyes-Ruiz y Surinach, 2015), la cual alude a la movilidad laboral de los científicos mexicanos hacia diversas partes del mundo, dada la falta de oportunidades laborales y la baja remuneración académica dentro del país.

La creación del SNI representaba la oportunidad de establecer una política que resultara en la permanencia de los investigadores mexicanos dentro del territorio, de tal forma que los productos de los investigadores trajeran beneficios que se reflejarían en competitividad económica para el país. La intención original de este programa de política pública se da claramente como consecuencia de un contexto económico mexicano golpeado por una crisis que ponía en juego la asignación de recursos a la investigación, además de condiciones de trabajo que no resultaban adecuadas para los investigadores, lo que los llevaba a buscar otros países para desarrollar su labor (Gérard, 2013; Rodríguez y Durand, 2013; Millán y Meza, 2015; López-Olmedo, et al, 2017).

A más de 30 años de su inicio, el SNI se presenta como un engrane importante de la estructuración de la ciencia en México (Didou y Gérard, 2011), ya que se ha vuelto un modelo a seguir por parte de toda la comunidad científica mexicana en todas las áreas de producción del conocimiento (Zubieta, et al., 1999). Sin embargo, hay posturas que analizan que la consolidación del SNI como eje crítico de la producción científica mexicana se ha dado a partir de la manera en que ha fungido como un mecanismo de consolidación de políticas neoliberales (Didou y Gérard, 2011); es decir, como el ensamble de una lógica sustentada en el control que ha permeado la vida académica (Ocampo-Gómez y Rueda-Hernández, 2015). Este discurso ofrece un importante punto de vista para la comprensión del papel del SNI en la vida académica del país cuando, a la par, se le considera como un elemento indispensable de la política impulsora de la producción científica de México (Reyes-Ruiz y Surinach, 2015).

\footnotetext{
$1 \quad$ En este trabajo, se utiliza el término "investigación científica" para referirse a aquellos trabajos orientados a la generación de conocimiento mediante un método y un proceso sistematizado, usualmente desarrollado bajo el cobijo de instituciones educativas de nivel superior o institutos de investigación públicos y privados, sin hacer diferencia alguna entre ciencias exactas, sociales, administrativas y humanidades.

2 El PNPC es un esfuerzo de la Secretaría de Educación Pública (SEP) y del Consejo Nacional de Ciencia y Tecnología (CONACYT) para agrupar a los programas educativos de posgrado que cubren ciertos criterios mínimos. Algunas de sus ventajas son el prestigio que el programa adquiere, además de la obtención de recursos para su operación (como becas para estudiantes de dedicación de tiempo completo).
} 
Frente a estos claroscuros, el presente manuscrito presenta una reflexión que pretende abonar al debate de la comprensión del papel del SNI como una parte de la política orientada a la producción científica en México. Este objetivo deviene de cuestionarse el papel de este programa en la generación de conocimiento científico, así como de valorar en qué se traduce la participación de tantos investigadores dentro del sistema. La relevancia del debate se da en función de su indiscutible papel en la vida académica de las universidades e Institutos de Educación Superior (IES) en México y en la vida misma de los académicos. Para aportar contenido a esta discusión se aborda un análisis bajo la mirada analítica de la técnica del poder (Han, 2014). La reflexión versa sobre la regulación del trabajo de los investigadores, previa exposición del origen y crecimiento del SNI desde su creación en 1984 hasta el año 2017. Finalmente, se analiza el papel de este programa en la implementación de la política del capitalismo académico como un agente del Estado Evaluador (Neave, 1988).

\section{Origen del SNI: programa orientado hacia la meritocracia}

El SNI se creó en México en los años ochenta y es operado por el Consejo Nacional de Ciencia y Tecnología (CONACYT) ${ }^{3}$ bajo un marco en el que la Secretaría de Hacienda y Crédito Público definió que los estímulos a la investigación tendrían que estar basados en el desempeño, sin obligaciones laborales a largo plazo; es decir, responsabilidades tales como los montos de pensión de los investigadores (Ricker, 2015). En el contexto de la crisis económica que México atravesaba, el SNI permitió hacer frente a la necesidad de aumentar los salarios de los investigadores (Reyes-Ruiz y Surinach, 2015), ofreciendo así una alternativa salarial como incentivo para mantener el desarrollo científico dentro del territorio nacional.

Este programa opera bajo una política del Estado mexicano orientada hacia la organización académica de los científicos del país. Su creación se estipuló por el Acuerdo Presidencial publicado en el Diario Oficial de la Federación el 26 de julio de 1984 (Silva, García y Aquino, 2016) y desde entonces, bajo ese importante marco normativo, ha fungido como un elemento de gran impacto en la vida académica del país.

El SNI opera a través de una convocatoria anual de participación voluntaria de los investigadores, fundamentado en la valoración de la productividad de los académicos en un periodo determinado. Como resultado, el CONACYT otorga un nombramiento con una vigencia en particular, además de un estímulo económico mensual asociado a este reconocimiento. Por medio de una revisión de la productividad de los investigadores, el SNI otorga un nivel de reconocimiento a los académicos en función de su productividad, a saber: Candidato, Nivel I, Nivel II, Nivel III y Eméritos. Los Eméritos son aquellos investigadores con al menos 65 años de edad y que han tenido al menos tres evaluaciones consecutivas como SNI III (además de tener quince años de manera ininterrumpida en dicho nivel). El nivel III es para los investigadores más experimentados, que tienen un alto número de doctorados finalizados; son los investigadores más reconocidos por sus publicaciones en las revistas más relevantes. Las diferencias entre los niveles I y II son la cantidad de trabajos publicados y los estudiantes titulados. El nivel Candidato es para recientes investigadores con menos de cinco artículos publicados (López-Olmedo, MarmolejoLeyva, et al., 2017).

Los montos de los incentivos económicos que se asignan al reconocimiento del SNI, así como la vigencia de cada nombramiento se han modificado con los años, pero en la convocatoria del 2017 se organizaron como se muestra en la Tabla 1. La intención del SNI de clasificar el nivel de los investigadores en función de la productividad es la de impulsar la formación de recursos humanos de alto nivel para la investigación, así como continuar otorgando apoyos para la generación de conocimiento científico y tecnológico de muy alta calidad (Contreras, et al., 2015; CONACYT, 2017b). De tal forma que el desarrollo de la investigación se promueva en función de la obtención de un mayor apoyo, dada una productividad en particular.

La participación de los académicos en el SNI es a través de siete áreas del conocimiento: I. Física, Matemáticas y Ciencias de la tierra; II. Biología, Guímica y Ciencias de la vida; III. Medicina y ciencias de la salud; IV. Humanidades y ciencias de la conducta; V. Ciencias sociales; VI. Biotecnología y ciencias agropecuarias; y VII. Ingenierías. En cada

3 EI CONACYT es un organismo público descentralizado del Gobierno Federal mexicano que tiene como fin promover y estimular el desarrollo de la ciencia. 
una de ellas existe una comisión dictaminadora (o evaluadora) que determina si las solicitudes de ingreso al sistema son aceptadas y también designan el nivel de cada investigador.

Así, el SNI pretende potenciar el desarrollo científico del país a través de la meritocracia; es decir, a través de la competencia entre académicos en función de su productividad. El nombramiento del SNI y el incentivo económico que se le asocia son productos del reconocimiento al esfuerzo individual de cada investigador para realizar investigaciones de calidad. La fundamentación de una política de fomento a la investigación basada en la competitividad o meritocracia está ligada a la restricción presupuestaria que la educación superior mexicana ha sufrido en las últimas décadas (Luna-Morales, et al., 2012), pero además ha permitido al Estado incrementar la competitividad en el ámbito de la ciencia, independientemente del presupuesto que reciban las instituciones que albergan a los investigadores y de las condiciones de jubilación de estos.

\section{Crecimiento del SNI entre 1984 y 2016: aceptación de los académicos de las intenciones competitivas del Estado}

El SNI ha sido altamente aceptado por la comunidad científica en México. Cuando inició, tuvo una participación de 349 investigadores (Contreras, et al., 2015) y al año 2016 contaba ya con 25,070 académicos dentro del sistema (CONACYT, 2017a).
La Figura 1 compara la cantidad de investigadores en niveles Candidato, I, II y III entre 1984 y 2016.

El incremento de participación en 32 años desde la creación de esta política es innegable. Si bien en la Figura 1 se observa claramente que la tendencia de contar con más investigadores en nivel I respecto a cualquier otro nivel permanece a pesar de los años, el éxito del CONACYT de fomentar el interés y la participación de los investigadores en esta política basada en la meritocracia es claro.

Pero ¿qué significa exactamente la generación de conocimiento científico? ¿En qué se traduce la participación de tantos investigadores dentro del sistema? Puede haber diversas perspectivas con respecto a lo que implica el término generación de conocimiento; sin embargo, el SNI tiene fijados sus criterios para valorar la competitividad y determinar el mérito de cada investigador. Un primer elemento es el nivel de preparación de los investigadores: quien desea ingresar al sistema debe contar con el grado de Doctor, salvo que se demuestre una producción y trayectoria académica muy relevante (Luna-Morales, et al., 2012). Cada comisión dictaminadora o evaluadora considera como valiosas las publicaciones de artículos, los libros o capítulos en libros dictaminados, la formación de profesionistas o posgraduados, actividades de docencia $\mathrm{y}$, en determinadas áreas de conocimiento, el registro de patentes. A pesar de esta amplia gama de productos por valorar, es bien sabido entre los miembros de la comunidad académica que el mayor impacto en la decisión de

Tabla 1

Tabulador de apoyos económicos a investigadores miembros del SNI 2017.

\begin{tabular}{lll}
\hline Nombramiento & Monto* & Vigencia del nombramiento \\
\hline Candidato & $\$ 6,800.00$ & Tres años \\
\hline I & $\$ 13,600.00$ & $\begin{array}{l}\text { Tres años en la primera distinción y cuatro años } \\
\text { más en una segunda designación }\end{array}$ \\
\hline II & $\$ 18,200.00$ & $\begin{array}{l}\text { Cuatro años en la primera distinción y cinco años } \\
\text { más en una segunda designación }\end{array}$ \\
\hline III & $\$ 31,900.00$ & $\begin{array}{l}\text { Cinco años en la primera y segunda distinciones y a } \\
\text { partir de la tercera designación consecutiva en este } \\
\text { nivel, la vigencia será de diez años }\end{array}$ \\
\hline Emérito & $\$ 31,900.00$ & Vitalicia \\
\hline
\end{tabular}

* Cifras expresadas en pesos mexicanos libres de impuestos.

Fuente: Acuerdo del nuevo reglamento del Sistema Nacional de Investigadores (DOF, 2017). 


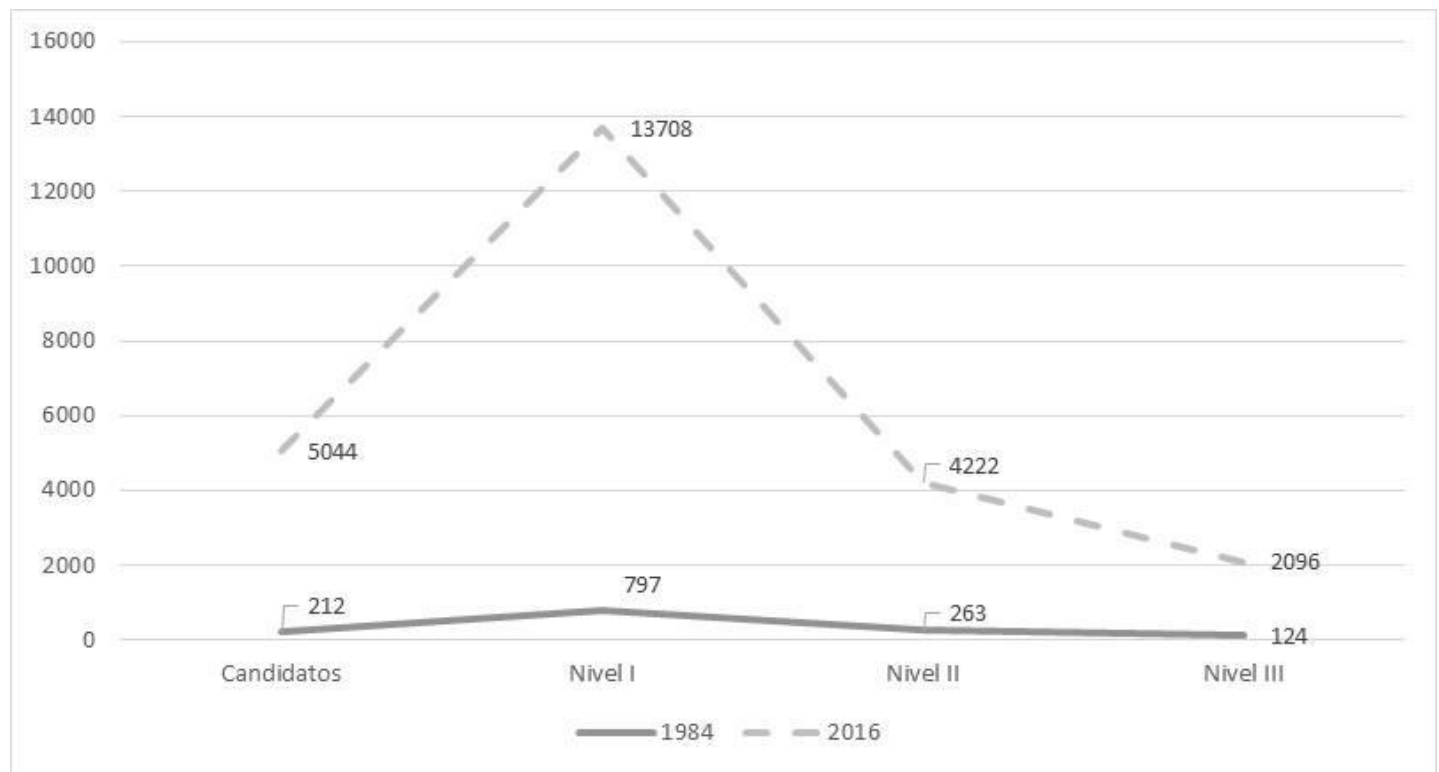

Figura 1. Número de investigadores pertenecientes al SNI entre 1984 y 2016.

Elaboración a partir de la información de Contreras, Baquero, et al., 2015 y CONACYT, 2017 a.

la asignación de un nivel en el SNI es el número de publicaciones, particularmente aquellas en revistas arbitradas e indizadas ${ }^{4}$ (Rodríguez y Durand, 2013).

$\mathrm{El}$ hecho que las publicaciones sean el elemento de mayor relevancia en el SNI responde a la intención meritocrática que fundamenta su implementación. La meritocracia se mantiene actualmente como el mecanismo indiscutible de otorgamientodesobresueldos, apoyosydeasignación de espacios laborales para los académicos. Esta forma de evaluación se fundamenta en la medición de determinados indicadores que, como se ha mostrado en otras investigaciones (CovarrubiasPapahiu, 2017), llevan a que las actividades que los investigadores realizan se planeen con la intención de generar puntuaciones para la obtención de estímulos como el SNI, dejando de lado la elaboración de estudios que no se consideren dentro del esquema meritocrático.

A pesar de esta consecuencia no deseada, la meritocracia que caracteriza al SNI ha sido eficiente en varios sentidos. El primero de ellos recae sobre los investigadores mismos. Como se observó en la Figura 1, el interés por ser parte del SNI es indudable y ha favorecido la formación de los profesionistas mexicanos, dado que ha orillado a la búsqueda de doctorados a personas de edades cada vez más tempranas, en virtud del requisito de tener este grado de estudios para ingresar al sistema (Didou y Gérard, 2011), permitiendo también acceder a un reconocimiento social como investigador nacional y a mejorar sus ingresos económicos.

En segundo lugar, la meritocracia en la cual se sustenta el SNI ha permitido que el Estado adquiera altos niveles de productividad científica que pueden ser sujetos de comparación a nivel internacional. Este logro no es menor, ya que esa búsqueda de competitividad -que se ha equiparado a la calidad de la investigación científica mexicanapretende ser un indicador del potencial de desarrollo económico del país. El cumplimiento de estos dos sentidos se ha dado sin necesidad de establecer compromisos salariales con los investigadores, más allá de un apoyo con una duración delimitada.

La eficiencia de la meritocracia y las ventajas que ha traído para ambos agentes (investigadores y CONACYT) se fundamenta en el prestigio que el SNI da tanto a los investigadores como al Estado en la medida en que se equipara con la calidad en el desarrollo científico de México. Independientemente del impacto que la ciencia producida en el país tenga en la mejora de las condiciones de vida de

$4 \quad$ La revisión entre pares favorece la confiabilidad del contenido de las revistas, mientras que la indización se ha establecido como una forma de impacto de las publicaciones. Algunos ejemplos son: Scopus, SCImago Journal Rank Indicator (JCR), índice h, o el Emerging Sources Citation Index (Vasen y Lunajo, 2017). 
la sociedad, en el desarrollo teórico que se genere o su aplicabilidad, la productividad fundamenta la lógica meritocrática que sustenta a este programa.

\section{La productividad y competitividad bajo responsabilidad de los académicos}

Hay dos tipos de logros dados por el SNI: el individual y el del Estado. Para los investigadores, la obtención de un nombramiento representa un reconocimiento de esfuerzos y años de trabajo académico. Para el Estado representa, por su lado, la obtención del nivel de competitividad científica deseada. En ambos casos se vive como una legitimación. Situándose desde los investigadores, y bajo la óptica del poder planteada por Han (2014), en esta búsqueda de legitimación individual, los académicos se vuelven trabajadores para sí mismos.

En el proceso de valoración y legitimación del investigador, el Estado no mantiene un papel de generador de condiciones de desarrollo de la función investigadora. Frente a la inestabilidad laboral, a la crisis financiera y a la demanda de las personas para acceder a condiciones dignas de trabajo, son los investigadores quienes se presionan a sí mismos para darle condiciones de bienestar al Estado. La meritocracia en la cual se fundamenta el SNI (además de otras formas de evaluación del trabajo académico ${ }^{5}$ ) es una muestra del mecanismo de poder (Han, 2014) en el cual los investigadores producen su trabajo bajo las exigencias de competitividad establecidas por el CONACYT sin necesidad de coerción alguna y sin responsabilidad por parte del Estado.

A partir de la reducción del financiamiento público a las IES, los investigadores y las universidades no han tenido más opción que facilitar la reestructuración de sus modos de financiamiento y gobierno (Walker, 2017). En aras de mantenerse laboralmente activos, los investigadores luchan por desarrollar su trabajo, no con el apoyo del Estado, sino a pesar de las limitaciones del Estado. En esa dinámica, la exigencia que el investigador se da a sí mismo para producir lo que el Estado desea se hace sin coerción o presión aparente; no se debe olvidar que la participación en el SNI es voluntaria.

El SNI apareció como una respuesta del Estado frente a un contexto de disminución del presupuesto para la educación superior y la investigación, así como de una desaceleración del crecimiento de la planta académica y, por supuesto, de una necesidad de establecimiento de regulación del trabajo de investigación (Buendía, et al., 2017). Bajo un panorama económico poco alentador para los investigadores, la respuesta del Estado para potenciar la competitividad fue el mecanismo meritocrático que traspasó la responsabilidad de la mejora salarial a los propios investigadores. En México, desde los años ochenta, el reconocimiento de la investigación y el apoyo a su desarrollo está en función de la evaluación de los méritos de los investigadores.

$\mathrm{Si}$, como analogía, se considera al Estado como el empleador y a los investigadores como los empleados (en términos dialécticos se les llamaría el amo y el esclavo), en este traspaso de responsabilidades laborales, ahora el empleado trabaja para el prestigio del empleador sin compromisos para este último. El esclavo trabaja ahora bajo su propio yugo sin ser consciente de su condición o, lo que es lo mismo, trabaja con base en la meritocracia. En términos hegelianos, el reconocimiento mutuo entre el esclavo y el amo permite que cada uno de ellos construya su identidad. Bajo este paradigma meritocrático del SNI, tal reconocimiento es inexistente, por lo que el poder de dominación del amo es absoluto, sin que el esclavo sea consciente de ello.

Este no es un asunto menor, ya que el Estado ha logrado la eficiencia para promover la investigación en México destinando recursos a la premiación de los logros de los académicos. Frente a la crisis, el SNI ofreció un estímulo para que los investigadores pudieran sustentar sus decisiones financieras personales (Contreras, et al., 2015), pero no implicó la inversión del Estado en más y mejores condiciones de trabajo académico, en la contratación de investigadores o en destinar mayores cantidades del Producto Interno Bruto (PIB) a la ciencia y la tecnologia ${ }^{6}$. El Estado no asumió la responsabilidad del desarrollo de la

$5 \quad$ El presente texto se enfoca en el SNI, que es el indicador en México que mide los avances en la investigación científica, aunque otras formas de valoración del trabajo académico se fundamentan en la misma lógica. Este tipo de trabajos pueden valorarse más a fondo en trabajos como los de Guzmán-Acuña y Martínez-Arcos (2015); Buendía, et al. (2017); Covarrubias-Papahiu (2017); y Walker (2017).

6 De acuerdo con datos del Banco Mundial (2017), en 2015 México destinó solo 0.5\% de su PIB a la ciencia y la tecnología cuando hay países como, por ejemplo, Israel y Estados Unidos, que destinan 4.27\% y 2.79\%, respectivamente. 
investigación, sino que transformó su función a la de un administrador de la política meritocrática que recargó en los investigadores la responsabilidad de producir el nivel competitivo esperado.

Por otra parte, el Estado -a través del CONACYT- ha dictado la agenda de la generación del conocimiento, de tal suerte que la búsqueda de la calidad (entendida como competitividad y prestigio) ha desplazado a la búsqueda del conocimiento en sí mismo y a la solución de problemas como elementos detonantes de una investigación. De esta manera, a través de la política meritocrática, la investigación se orienta hacia las líneas y formas de trabajo que se ven favorecidas por la productividad aceptada por el canon establecido por el CONACYT (ReyesRuiz y Surinach, 2015).

En resumen, la eficiencia de la meritocracia del SNI y el poder que tiene sobre la investigación no solo se ven reflejados en el crecimiento del número de investigadores entre 1984 y 2016, sino sobre todo en la responsabilidad que los investigadores han asumido como agentes responsables del desarrollo del Estado, además de que ahora las líneas de generación del conocimiento son aquellas que potencian la competitividad económica del país. Como lo afirma Ricker (2015), la consecuencia de esta dinámica es que los investigadores se orientan a servir al algoritmo más que a la sociedad misma.

\section{El SNI como parte de los mecanismos del Estado Evaluador}

El establecimiento de la meritocracia como un mecanismo aceptable para el establecimiento de apoyos a la investigación reside en una base a la que Neave (1988) llamaría ultraliberalista, que ha caracterizado al establecimiento del Estado Evaluador. Es decir, frente a un Estado que no proporciona condiciones de trabajo para el desarrollo de la investigación, la regulación administrativa de la población académica, a través de la meritocracia, permite una alta productividad en investigación. Bajo un enfoque ultraliberalista el Estado mexicano se apoya en la evaluación de la productividad de los académicos como medio de control sobre la investigación.

El concepto de Estado Evaluador refleja la intención del Estado de regular y controlar el desarrolloacadémico; en estecaso, delainvestigación por medio de mecanismos que le permitan fungir más como un agente de administración de los recursos limitados, que como un elemento que potencie el desarrollo en todos sus ámbitos. Esa transición ofrece una primera mirada de aparente no intervención sobre los procesos de investigación; sin embargo, el énfasis en la evaluación refuerza el control que el Estado tiene ahora sobre la vida académica. Este control se da dentro de los límites de acción que los agentes educativos tienen, los cuales han sido delimitados por el Estado mismo (Neave, 1988).

En México, la evaluación en el trabajo académico se implementó como política de Estado como parte del Programa de Modernización Educativa en los años ochenta y se ha mantenido en el transcurso de los programas de política federal de los últimos sexenios (el Programa de Desarrollo educativo -1995 a 2000-, el Programa Nacional de Educación -2001 a 2006- y el Programa Sectorial de Educación -2007 a 2012-) (Buendía, et al, 2017). Esta implementación se encuentra más sólida que nunca y el Estado Mexicano ha adquirido, cada vez más, un papel como agente en la administración y regulación del financiamiento a la investigación más que como un benefactor de su desarrollo (Walker, 2017).

Este giro administrativo o de regulación del Estado sobre la vida de la investigación científica ha facilitado la implementación de un capitalismo académico (Walker, 2017) que asume que las prácticas académicas se transformen bajo la lógica de una empresa. La idea central del capitalismo académico en la investigación es que todos los desarrollos se centran en aquellos elementos que permitan el aseguramiento de ingreso y mantenimiento en el SNI a quienes demuestren ser parte del mecanismo de poder meritocrático, que se traducirá en capital incorporado y objetivado (Ocampo-Gómez y Rueda-Hernández, 2015). De tal suerte que este énfasis del SNI en la evaluación de la investigación y su influencia para favorecer la realización de tareas investigativas puede estar en duda, ya que, como lo comentan Rodríguez y Durand (2013), el SNI tradicionalmente se ha enfocado en premiar a quienes cumplen con estándares fijos establecidos en lo que CONACYT entiende como generación de conocimiento.

Esta implementación del capitalismo académico que subyace a las ideas de premiar a las actividades de investigación por medio de prestigio e incentivos económicos, permite afianzar la idea de calidad establecida por el Estado Evaluador (Di- 
dou y Gérard, 2011). Esta perspectiva de calidad a través de la competitividad ha sido un elemento de control, la cual se establece como mínimo ideal que los investigadores deben alcanzar para mantenerse dentro del sistema. En el imaginario cotidiano, la calidad de la investigación mexicana se comunica a la sociedad a través del SNI, de manera que los investigadores que logran tal reconocimiento se legitiman como un agente incuestionable de desarrollo de la ciencia (Contreras, et al., 2015). Sin embargo, ¿cómo es que se define esa calidad? ¿Quién define las formas de legitimación de los investigadores como agentes de investigación de calidad?

La valoración de la inclusión de un investigador dentro del SNI se realiza a través de las ya nombradas comisiones dictaminadoras o evaluadoras, que están conformadas por investigadores de gran renombre y trayectoria en cada una de las áreas del conocimiento en las cuales se organiza el SNI. Los miembros de las comisiones son investigadores con, al menos, nivel III del SNI. De esta manera, son los investigadores más reconocidos de cada campo del conocimiento quienes reproducen la idea de calidad de la investigación como competitividad (al igual que la idea del capitalismo académico) premiando con reconocimiento e incentivos a los colegas que han desarrollado los productos académicos que el Estado promueve. Así, son los académicos quienes fungen como brazo del Estado Evaluador.

Como paréntesis, y recordando que los productos mayormente valorados dentro del SNI son las publicaciones dentro de revistas arbitradas entre pares, son estas revistas los agentes determinantes para valorar la calidad de la productividad académica (Vasen y Lunajo, 2017). De esta forma, las lineas editoriales que pueden responder a intereses públicos o particulares (dependiendo del tipo de institución que las albergue) pueden definir la agenda de investigación. No es que las publicaciones generen en sí mismas un ingreso a los investigadores, sino que su elaboración establece un capital que favorece su inclusión o mantenimiento dentro del SNI. De esta manera, al igual que como se comentaba previamente, las líneas de investigación pueden verse influidas más por la búsqueda de la dinámica del capitalismo académico que por las necesidades propias de la investigación y el conocimiento.

Es a través de la valoración de las comisiones dictaminadoras que el Estado Evaluador legitima la implementación de condiciones de trabajo ultraliberalistas a los académicos. La evaluación realizada por los académicos a los productos desarrollados de un investigador determinará el prestigio que se obtendrá, que al ser ya un capital del evaluado, puede ayudarle a obtener fuentes de financiamiento para seguir desarrollando investigaciones (Luna-Morales, et al., 2012). Esta cadena de evaluación, que conlleva al apoyo salarial y posteriormente a la obtención de un prestigio o legitimación, permite que quienes participen en el SNI generen cada vez más posibilidades de mantenerse en él. Esto acarrea problemas de una planta de investigadores envejecida (Didou y Gérard, 2011) que no busca la jubilación en aras de no abandonar la posibilidad de un capital importante. Siguiendo la analogía hegeliana previamente utilizada, no ven la posibilidad de renuncia de ser esclavos de sí mismos.

El Estado ha buscado en la investigación uno de sus soportes de competitividad frente a la apertura económica mundial (Walker, 2017). El SNI ha fungido por más de treinta años como un brazo importante de la política evaluadora del Estado mexicano, que a través del discurso de calidad y de prestigio ha sido un paliativo del problema de bajos salarios que el mismo Estado promueve. De esta forma, los investigadores han forjado un engranaje importante en la competitividad deseada por el Estado Evaluador, que ha encontrado en esta herramienta un mecanismo idóneo para regular la formación de jóvenes doctores y gestionar la producción de artículos científicos en revistas de reconocido prestigio sin necesidad de acrecentar la inversión en la educación superior ni mejorar las condiciones de trabajo o el desarrollo de la investigación.

\section{Conclusiones}

El poder que el Estado ha ejercido sobre la investigación cientifica ha incrementado desde 1984. La manera en que ha operado ha sido relativamente amable, más que represiva (Han, 2014). Desde hace más de treinta años, el SNI ha abierto la posibilidad de que exista una mayor formación de jóvenes en la investigación y que los investigadores en activo se consoliden a través de la meritocracia y se reconozcan socialmente como un motor importante en la competitividad de México. Esa libertad de formación y de posibilidad de legitimación de los investigadores por parte del 
Estado difiere de un poder disciplinario basado en coerciones al gremio académico. Es esta libertad de legitimación y amabilidad en el poder las que caracterizan al SNI como no impositivo, haciéndolo aún más poderoso (Han, 2014).

La meritocracia que subyace a este programa de política pública seduce a quienes quieren ser investigadores o quienes pretenden consolidarse, ya que ofrece la posibilidad de desarrollar investigación científica, y a la par, solucionar la problemática de los malos salarios y pocas oportunidades de desarrollo que el Estado ofrece. En ese sentido, el CONACYT apoya al Estado mexicano en ofrecer un rostro de posibilidad de crecimiento y consolidación para los investigadores, aunque el papel del Estado realmente solo se esté limitando al establecimiento de categorías para supervisar el cumplimiento de la producción esperada.

Es justo en este papel de establecimiento de criterios de evaluación o supervisión que el CONACYT favorece la consolidación del Estado Evaluador en México. Es mediante ese mecanismo amable que el poder del Estado Evaluador promueve que los investigadores no se conciban como agentes de desarrollo social del Estado Mexicano sino como sujetos de observación. La utilidad de la investigación científica en el beneficio de la humanidad es, bajo cierta lógica, incuestionable; sin embargo, el trabajo de investigación se ve fuertemente influido por la supervisión que ejerce el Estado.

Más que considerar esto como una crítica a una mala práctica del ejercicio de investigación, la intención del presente manuscrito es aportar elementos de discusión del papel del SNI frente a los procesos del capitalismo académico que se viven en las IES mexicanas. Se espera así, que la comprensión del SNI bajo la mirada presentada apoye las actuales corrientes de académicos que discuten las formas de evaluación del trabajo académico, como en los trabajos de Rip y Van der Meulen, (1996); Levin, (2004); Didou y Gérard, (2011); Rodríguez y Durand, (2013); Ocampo-Gómez y Rueda-Hernández, (2015); y Walker, (2017).

Frente a esta situación, más que cuestionar los algoritmos y las formas de evaluación del trabajo académico (los textos de Ricker (2015) y Buendía, et al., (2017) tienen un análisis profundo sobre ello), es importante valorar la manera en que el SNI -y sus académicos dentro de las comisiones dictaminadoras- está fomentando el establecimiento de un poder que limita la identificación dialéctica entre los actores involucrados, nublando el hecho de que los académicos están fungiendo como esclavos, no de un amo ajeno a ellos, sino de sí mismos bajo una cara amable del poder.

En ese sentido, ¿qué opciones se tienen frente a esa problemática desde la vida de la investigación científica (en todas sus áreas de conocimiento)? ¿Es factible no ser parte del SNI? ¿Es deseable no serlo? Pareciera que el debate no debe versar nuevamente sobre los académicos y sus decisiones de pertenencia al SNI o no, sino que el análisis puede apuntalarse en el papel del Estado Mexicano frente a su responsabilidad como soporte de desarrollo de la ciencia y la tecnología en el país. El Estado, a través del CONACYT, no debe fungir solo como medio regulador del poder del capitalismo académico, sino como factor de desarrollo de las condiciones necesarias para realizar investigación de impacto. Una propuesta en este sentido podría traer más beneficios a largo plazo para toda la sociedad mexicana que solo pensar en incentivos, prestigio y legitimación.

\section{Referencias}

Banco Mundial (2017). Gasto en investigación y desarrollo. Recuperado de https://datos. bancomundial.org/indicador/GB.XPD.RSDV. GD.ZS?view=chart [21 de diciembre de 2017].

Buendía, A. et al. (2017). Queríamos evaluar y terminamos contando: alternativas para la evaluación del trabajo académico. Perfiles educativos, 39(157), pp. 200-219.

Cabrero-Mendoza, E. (2015). Principales logros y desafíos del Sistema Nacional de Investigadores de México a 30 años de su creación. Revista Iberoamericana de Ciencias, Tecnología y Sociedad, 10(28), pp. 1-12.

Consejo Nacional de Ciencia y Tecnología (CONACYT) (2017). Sistema Nacional de Investigadores. Convocatoria 2017. Investigadores Nacionales Eméritos. Recuperado de http://www.conacyt. gob. mx/index.php/sni / convocatoriasconacyt/convocatorias-sistema-nacional-deinvestigadores-sni/convocatorias-abiertassni/inv-emeritos / 14314 -convocatoriainvestigadores-nacionales-emeritos-2017/file [12 de diciembre de 2017].

Contreras, L. et al. (2015). Patrones de movilidad de los físicos mexicanos en el Sistema Nacional de Investigadores. Interciencia, 40(8), pp. 525532 .

Covarrubias-Papahiu, P. (2017). Situación actual de los 
programas de evaluación académica de la educación superior mexicana: sus efectos en el trabajo académico. Revista Iberoamericana de Evaluación Educativa”, 10(2), pp. 187-209.

Diario Oficial de la Federación (DOF) (2017). Acuerdo con el que se emite el nuevo Reglamento del Sistema Nacional de Investigadores. Recuperado de http://www.dof.gob.mx/nota_detalle. php?codigo $=5470107 \&$ fecha $=27 / 01 / 2017 \quad[1$ de diciembre de 2017].

Didou, S. y Gérard, E. (2011). El Sistema Nacional de Investigadores en 2009. ¿Un vector para la internacionalización de las élites científicas?. Perfiles educativos, 33(132), pp. 29-47.

Gérard, E. (2013). Dynamiques de formation internationale et production d'élites académiques au Mexique. Revue d'anthropologie des connaissances, 7(1), pp. 317-344.

Guzmán-Acuña, T. y Martínez-Arcos, C. (2015). The effectiveness of Mexico's Faculty Improvement Program (PROMEP) in Public State Universities. Education Policy Analysis Archives, 23(55), pp. $1-26$.

Han, B.-C. (2014). Psicopolitica. Barcelona: Herder.

Levin, B. (2004). Making research matter more. Education Policy Analysis Archives, 12 (56), pp. 1-22

López-Olmedo, R. et al. (2017). The role of public policies in the descentralization process of Mexican science and the formation of new researchers in institutions outside Mexico City area. Scientometrics, (112), pp. 1343-1366.

Luna-Morales, E. et al. (2012). Evolución e impacto de la investigación en la Universidad Autónoma de San Luis Potosí, México. Patrones de publicación y Sistema Nacional de Investigadores. Bibliotecología, 26(58), pp. 175213.

Márquez, A. (2010). Estudio comparativo de universidades mexicanas (ECUM): otra mirada a la realidad universitaria. Revista Iberoamericana de Educación Superior, 1(1), pp. 148-156

Millán, G. y Meza, N. (2015). Los miembros del Sistema Nacional de Investigadores mexicano: un acercamiento desde la producción de patentes 2003-2012. Interciencia, 40(12), pp. 840-846.
Neave, G. (1988). The evaluative state reconsidered. European Journal of Education, 33(3), pp. 265284.

Ocampo-Gómez, E. y Rueda-Hernández, J. (2015). El Sistema Nacional de Investigadores en la Universidad Veracruzana: análisis exploratorio de cómo se experimenta el reconocimiento académico. Revista Interamericana de Educación de Adultos, 37(1), pp. 64-85.

Reyes-Ruiz, G. y Surinach, J. (2015). Análisis sobre la evolución del Sistema Nacional de Investigadores (SNI) de México. Investigación administrativa, (115), pp. 55-69.

Ricker, M. (2015). A numercial algorithm with preference statements to evaluate the performance of scientists. Scientometrics, (103), pp. 191-212.

Rip, Ay Van der Meulen, B. (1996). El sistema de investigación posmoderno. Redes, 3(6), pp. 13-31.

Rodríguez, J. y Durand, J. (2013). Notas para la evaluación del trabajo académico. Perfiles educativos, (35) especial, pp. 46-56.

Silva, M., García, V. y Quino, S. (2016). Retos del crecimiento del Sistema Nacional de Investigadores (SNI) del Consejo Nacional de Ciencia y Tecnología (CONACYT) en México. Actualidades Investigativas en Educación, 16 (2), pp. 1-24.

Vasen, F., y Lunajo, I. (2017). Sistemas nacionales de clasificación de revistas científicas en América Latina: tendencias recientes e implicaciones para la evaluación académica en ciencias sociales. Revista Mexicana de Ciencias Politicas y Sociales, 42(231), pp. 199-228.

Walker, V (2017). La evaluación como mecanismo de regulación del trabajo académico. Estudios de casos en universidades de Argentina y España. Archivos Analíticos de Políticas educativas, 25(108).

Zubieta, J. et al. (1999). Problemática del desarrollo científico y tecnológico en México. Estudios mexicanos, 15(1), pp. 193-211. 